\title{
Environmental High Frequency Characterization of Fabrics based on a Novel Surrogate Modelling Antenna Technique
}

\author{
Frederick Declercq, and Ivo Couckuyt, and Hendrik Rogier Senior Member IEEE, and Tom Dhaene senior \\ Member IEEE
}

\begin{abstract}
Wearable antennas are mostly constructed from fabric or foam, whereas e-textiles are often used as conductive parts. A design obstacle is the lack of knowledge about the electromagnetic properties of these materials. Moreover, most of these fabrics exhibit electromagnetic properties that depend on prevailing atmospheric conditions. In this work, we present a dedicated characterization method to determine the complex permittivity of fabrics or foams, as well as the effective conductivity of e-textiles, and this as a function of relative humidity. The method extracts the constitutive parameters by comparing measured and simulated antenna figures of merit such as input impedance and antenna efficiency. This inverse problem is solved using a surrogate-based optimization technique as implemented in the SUrrogate MOdeling Toolbox, yielding a fast and accurate characterization. The method is evaluated by characterizing six materials which are exposed to relative humidity levels ranging from $10 \%$ to $90 \%$. From the extracted complex permittivities of the six materials, two-phase dielectric mixing models based on the volumetric fractions of the absorbed moisture in the substrates are developed and evaluated in terms of accuracy. For the materials exhibiting a high sensitivity to moisture, the model is observed to be less accurate. However, the worst model accuracy is shown to be comparable with the estimated accuracy of the characterization procedure. For materials with low sensitivity to moisture, the model fits the measured values very well.
\end{abstract}

Index Terms-permittivity, loss tangent, conductivity, e-textile, relative humidity, kriging, microstrip antennas, textile antennas

\section{INTRODUCTION}

$\mathbf{I}$ $\mathrm{N}$ the last decade numerous textile planar antennas for wearable applications were designed and reported in literature [1]-[7]. Off-the-shelf fabric or foam materials typically serve as the antenna substrate, whereas e-textiles are often used as conductive parts. Also printing technology and flexible polyimid/Cu sheets have been applied in defining conductive patterns onto a fabric dielectric [8], [9]. Adopting these flexible materials in wearable antenna design fulfills the requirement of conformability to the human body and facilitates the integration of wearable textile systems into the garment.

Manuscript received August 8, 2012; revised March 21, 2013; accepted July 1, 2013. Date of publication Date, year.

Frederick Declercq and Hendrik Rogier are with IMEC, INTEC division. They are also affiliated with the Department of Information Technology, Ghent University, St. Pietersnieuwstraat 41, Ghent, Belgium. E-mail: frederick.declercq@intec.UGent.be, Tel.: ++32/92649531, Fax: ++32/92649969.

Ivo Couckuyt and Tom Dhaene are with the Department of Information Technology, Ghent University, iMINDS, Gaston Crommelaan 8, 9050 Ghent, Belgium
The introduction of fabrics in wearable antenna design initiated the need for accurate electromagnetic property characterization of the non-conductive substrate material as well as the conductive fabrics. Several microwave methods have been adopted in the characterization of textile fabrics. These methods can be split into two groups, being the non-resonating and resonating methods. The former group, such as the broadband transmission line method, utilizes scattering parameter measurements of two textile microstrip lines with different lengths in order to extract the material's permittivity [10], [11]. This transmission line technique was improved by using the matrix-pencil method which minimizes the deviation of the extracted dielectric parameters [12]. The second group are the resonating methods. Compared to the non-resonating methods, the latter group is narrow band but provides a higher accuracy and sensitivity [13]. Moreover, the cavity perturbation technique also identifies anisotropy that is typically present in the electromagnetic properties of woven textile materials [2], [11]. Finally, planar antenna resonator techniques are commonly used because of their simple sample preparation. Moreover, they characterize the fabrics as in the final application, taking into account the effect of glue and e-textile [12]. In general, permittivity is extracted by comparing the measured antenna resonance frequency with the simulated resonance frequency based on full-wave numerical techniques such as the Method of Moments [14] or with the resonance frequency calculated using empirical formulas [15]. For the characterization of conductive yarns, the waveguide cavity and transmission line methods were used in [16] and [17], respectively. Conductive fabric characterization by means of a microstrip-resonator method and a transmission line method were addressed in [17] and [18], respectively.

Since wearable antennas operate in a continuously changing environment, a thorough understanding of the antenna behavior as a function of environmental conditions is absolutely essential. These environmental conditions are realized in climate chambers in which relative humidity and temperature are accurately controlled. Only few efforts focused on changing antenna performance and electromagnetic properties of the materials as a function of environmental conditions. In [14], the relative permittivity of several antenna substrates, exhibiting different sensitivities to moisture, as a function of relative humidity was determined. Quantitative environmental characterization of the substrate's complex permittivity using a transmission line and a cavity perturbation method is presented 
in [11], [19]. A good overview of the dielectric properties of textile materials and fibres, and the various low frequency and RF measurement techniques is given in [20]. In [21] qualitative experiments were performed on different textile antennas, characterizing their reflection coefficient behavior and their in-system performance when exposed to water, ice and snow.

In this work, we propose a novel electromagnetic property characterization method dedicated to characterization of antenna materials as a function of varying relative humidity. The method relies on a surrogate-based optimization (SBO) approach for minimizing a cost function that quantifies the difference between measured and modeled antenna figures of merit and hence reconstruct complex permittivity, being permittivity and loss tangent of the substrate, and conductivity of the e-textile. The SBO technique for dielectric characterization of substrates by solely relying on antenna reflection coefficient was introduced in [22]. In this work, we improve the accuracy of this technique by incorporating antenna efficiency in the new characterization process. Furthermore, a dedicated fast measurement procedure is introduced as antenna measurements are infeasible in a climatic chambers. Furthermore, we used the measured substrate data to investigate the applicability of a dielectric mixing model. In particular, the Birchak mixing model based on volumetric fractions is used.

Converting the inverse problem into a forward optimization process is a popular approach in material characterization. In [23] the dispersive dielectric properties of PCB substrates were determined by using a genetic algorithm for minimizing the cost function. In [24], a longitudinally inhomogeneous dielectric profile inside a metallic waveguide was reconstructed by relying on different Gauss-Newton techniques. A technique for characterizing dielectric properties of biological tissues was presented using a rational function model (RFM) of an immersed open-ended coaxial probe in [25]. A matlab optimizer minimized the cost function between the measured and RFM aperture admittance. In [26] subsurface electric properties of soils were reconstructed using a model of a ground penetrating radar that includes the multilayered soil surface. The inverse problem was solved by relying on an efficient global optimization scheme. Also neural networks [27], [28] and space mapping [29] techniques were used for solving inverse electromagnetic problems for finding geometric and electromagnetic properties.

Section II describes the two-step characterization procedure. In Section III, the constitutive parameters of a high frequency laminate with known parameters are determined in order to validate the method's accuracy. The six different fabric materials characterized in this work and the measurement setup for investigating conditioned antenna figures of merit is given in Section IV. Next, the results of the characterization process are presented in Section $\mathrm{V}$ and the need for the improved cost function is highlighted. Finally, in Section VI, we apply a dielectric mixing formula onto the results of the characterization process and evaluate the accuracy of the characterization method and the mixing model.

\section{Characterization Process}

\section{A. Electromagnetic Properties of Fabrics}

The electromagnetic properties of interest are complex permittivity $\epsilon=\epsilon_{0} \epsilon_{r}(1-j \tan \delta)$ of the wearable antenna substrate, with $\epsilon_{0}=8.85 \times 10^{-12} \mathrm{Fm}^{-1}$ being the free space permittivity, $\epsilon_{r}$ representing the relative permittivity and $\tan \delta$ denoting the loss tangent of the substrate. In addition, when applying e-textiles in wearable antenna design, the conductivity $\sigma$ is of interest for the antenna design engineer. Note that dielectric substrate losses will be included in the complex $\epsilon$, whereas $\sigma$ accounts for the conductive losses in antenna and ground planes. Wearable antennas are constructed from fabric or foam material which are heterogeneous mixtures of three different constituents, being textile fibres or bulk foam material, air and moisture. Furthermore, fibrous assemblies such as woven fabrics also exhibit an anisotropic behavior [19] and the complex permittivity depends on temperature. In the heterogenous fibre assembly, the individual permittivities of air, fibre and moisture contribute to the overall $\epsilon$ of the substrates and can be estimated by relying on dielectric mixing laws [30]. However, the structure of non-woven fabrics and foams is highly irregular which makes the prediction of dielectric properties as a function of geometrical parameters and the dielectric properties of the individual constituents highly problematic. Despite the fact that woven fabrics consist of a quasi-periodic repetition of unit structures, geometrical modeling is still inconvenient due to variation in yarn geometry, variation among the individual yarns and variations due to the weaving process. In the considered frequency range of the characterization process, the size of the moisture and air inclusions is small with respect to the wavelength allowing us to homogenize the substrate and apply dielectric mixing models that use fractional volumes, which can be easily measured, as a parameter relating the permittivities of the individual constituents in the overall permittivity [30]. This approach is also well known to describe the behavior of wet soil [31].

In general complex $\epsilon$ depends on frequency and the simplest model for describing the dispersive behavior of a non magnetic, linear, isotropic and homogenous material is given by the Debye law involving a single relaxation mechanism [23]. Due to the presence of water in the substrates, more than one relaxation mechanism will exist and the overall dispersive behavior of the substrate has to be described by a model that sums two Debye models with different relaxation mechanisms [26]. Note that for water, both dielectric losses due to polarization of the water molecules and conductive losses due to ionic conductivity contribute to the overall $\tan \delta$ of the antenna substrate. For the frequency range considered in this study, the losses due to relaxation of the water molecules start to prevail, resulting in an increase of the dielectric losses. This work presents a narrowband characterization technique allowing us to consider the complex $\epsilon$ as being constant. Furthermore, we assume isotropic behavior of the substrates since the main field component for antenna radiation in planar antenna design is the out-of-plane component [32]. 


\section{B. Characterization Method}

The reflection coefficient $S_{11}(f)$ and antenna radiation efficiency $e_{c d}(f)$ of a planar antenna, with $f$ being the frequency, are determined by the complex $\epsilon$ of the substrate and $\sigma$ of the conductive plane. The constitutive properties of all materials used in a planar antenna can be determined by fitting simulated onto measured antenna figures of merit. This inverse problem is solved by converting it into a forward optimization problem [22]. Practically, a full-wave electromagnetic simulation model of the antenna is constructed and the constitutive parameters in the substrate model are optimized until a minimum is found in the cost function that quantifies the difference between the simulated and measured antenna performance characteristics. An SBO technique was chosen for the minimization of the cost functions as SBO outperforms other blackbox optimizers such as Simulated Annealing or Pattern Search in terms of accuracy and optimization speed [22]. The simulation model of the antenna, the two different cost functions and the SBO algorithm are discussed in this section. In order to find a unique solution, the characterization process is divided into two sub-characterization processes:

- Step 1: We first characterize a planar antenna constructed using homogeneous copper foil with a known $\sigma$ for the conductive planes. The outcome of the characterization process yields the substrate's $\epsilon_{r}$ and $\tan \delta$.

- Step 2: An e-textile based antenna constructed using the same dielectric material as in step 1 is characterized in step 2. Reusing the extracted $\tan \delta$ from step 1 and optimizing for $\sigma$ and $\epsilon_{r}$ to minimize the cost function yields the remaining constitutive parameters of all materials needed to accurately design the textile antenna.

Note that in step 2, $\epsilon_{r}$ is still unknown due to the fact that resonance frequency decreases when applying e-textiles in stead of copper foil, for the conductive parts in wearable antenna design [12]. One can take this effect into account by using an equivalent substrate $\epsilon_{r}$ in the simulation model. This equivalent permittivity is slightly larger than the $\epsilon_{r}$ in the simulation model of the copper based antenna design. Practically, the substrate parameters and conductivity are constrained to a prescribed optimization range. These bounds depend on the materials' susceptibility to moisture and on the initial estimate for the material properties used in the textile antenna designs. Furthermore, the optimization range of the substrate's $\epsilon_{r}$ is chosen such that we avoid higher-order mode resonances of the planar antenna, which could potentially result in multiple solutions of the sub-characterization process.

1) Simulation Model of the Antenna: The antenna model relies on an integral equation solved by the Method of Moments as implemented in Momentum from Agilent Technologies' Advanced Design System. The antenna topology used in the characterization process is an inset-fed patch antenna as depicted in Fig. 1. The following geometrical assumptions were made in the simulation model of the antennas:

- The ground plane in the simulation model is of infinite size.

- The SMA connector connected to the antenna feedline is not accounted for in the simulation. We use a single port

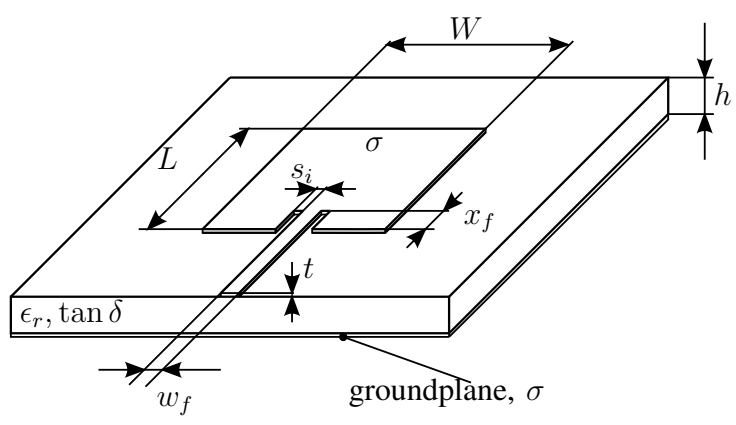

Fig. 1. Geometry of the inset-fed patch antenna.

excitation in the simulation model in which a calibration procedure removes potential higher order mode excitations and port excitation parasitics in the S-parameter calculation of the antenna [33].

- The uncertainty in geometrical antenna parameters is not considered. Predominantly, uncertainties in the length $L$ of the patch and thickness $h$ of the substrate yield the largest uncertainty in the outcome of the characterization process. Compressibility of the flexible substrates and increase in substrate thickness due to water absorption are the main contributors to the uncertainty in $h$. The finite fabrication accuracy of the patch determines the uncertainty in $L$.

To simplify the characterization process, we neglect changes in actual thickness due to moisture, but we incorporate this effect in the equivalent $\epsilon_{r}$ of the substrate, keeping substrate thickness constant in the simulation model.

The substrate model in Momentum implies a homogeneous and isotropic substrate. Hence, $\epsilon$ of the substrate model incorporates the individual contributions of the constituents (bulk material, air, water) and the frequency dependence of the substrate's complex permittivity due to the air-dielectric interface is accounted for by means of the full-wave Dyadic Green's function [33]. The e-textile is modeled as a homogeneous conductor with an effective thickness and an equivalent conductivity yielding the same losses as introduced by the e-textile. The effective thickness was chosen to be identical to the physical e-textile thickness. The frequency dependent conductivity model was chosen to model conductor losses.

2) Cost Functions: Since $f_{r}$ and $B W$ are captured in the antenna's reflection coefficient curve as a function of frequency, one can extract substrate $\epsilon_{r}$ and $\tan \delta$ in step 1, or substrate $\epsilon_{r}$ and conductor $\sigma$ in step 2 by fitting simulated onto measured reflection coefficients. The cost function describing the discrepancy between simulated and measured data is the popular mean squared error as applied in [22],

$$
M S E_{1}=\frac{1}{n} \sum_{i=1}^{n} w_{i}\left(\left|S_{11, i}\right|^{d B}-\left|\widetilde{S_{11, i}}\right|^{d B}\right)^{2}
$$

with $n$ the number of frequency points, and $\left|S_{11, i}\right|$ and $\left|\widetilde{S_{11, i}}\right|$ the reflection coefficients of the antenna at frequency $i$ obtained by simulation and measurements, respectively. $w_{i}$ is the weighting factor for the simulated and measured reflection 
coefficient at frequency $i$. It is well known that the real part of complex permittivity has a more significant influence on resonance frequency compared to $\tan \delta$. Hence, (1) is quickly minimized if an approximation of the true substrate's $\epsilon_{r}$ is known. Subsequently, further exploration of $\epsilon_{r}$ and $\tan \delta$ (step 1) or $\sigma$ (step 2) near the approximate $\epsilon_{r}$ until both resonance curves $\left|S_{11}\right|$ and $\left|\widetilde{S_{11}}\right|$ coincide yields the correct $\left(\epsilon_{r}, \tan \delta\right)$ in step 1 or $\left(\epsilon_{r}, \sigma\right)$ in step 2 of the characterization process. As shown in Section III and IV, the sensitivity of the characterization process to variation in loss tangent of the substrate is relatively poor. This will result in an inaccurate extraction of substrate $\tan \delta$ in step 1, which increases the uncertainty of the simulation model in step 2 of the characterization process. Therefore, an improvement to (1) in terms of sensitivity to variations in $\tan \delta$ is proposed by also incorporating antenna radiation efficiency in the cost function. The new cost function is then given by

$$
\begin{array}{r}
M S E_{2}=a_{1} \frac{1}{n} \sum_{i=1}^{n} w_{i}\left(\left|S_{11, i}\right|^{d B}-\left|\widetilde{S_{11, i}}\right|^{d B}\right)^{2} \\
+a_{2}\left|e_{c d, f_{r, s}}-\widetilde{e_{c d}, f_{r, m}}\right|,
\end{array}
$$

where $e_{c d, f_{r, s}}$ and $\widetilde{e_{c d}, f_{r, m}}$ are the simulated and measured antenna efficiencies at the simulated and measured resonance frequencies $f_{r, s}$ and $f_{r, m}$, respectively. The weighting factors for the two individual parts of the error function are represented by $a_{1}$ and $a_{2}$. Note that $\left|e_{c d, f_{r, s}}-\widetilde{e_{c d}, f_{r, m}}\right|$ is not squared since the order in magnitude of the efficiency error is similar to the order of magnitude of the first error term when choosing $a_{1}=1$ and $a_{2}=100$. The weighting factors $w_{i}$ in (1) or (2) equal 1 for all frequencies $i$.

3) Characterization Flowchart: A schematic overview of the complete characterization process is given in Fig. 2. First, an estimated value for the electromagnetic properties $\left(\epsilon_{r}, \tan \delta\right)$ is utilized in Momentum from Agilent Technologies in order to design an inset-fed patch antenna as depicted in Fig. 1. The use of a single-mode narrow bandwidth antenna increases the sensitivity of the resonance frequency with respect to small perturbations in $\epsilon_{r}$ yielding a more accurate characterization. From this design, a simulation model, in which the input variables are the materials' electromagnetic properties, is constructed in Momentum. The outputs of the simulation model are $\left|S_{11}\right|$ and $e_{c d}$ at $f_{r}$. Two textile antennas for each substrate are required: One prototype utilizing homogeneous $\mathrm{Cu}$ foil and one using e-textile for the conductive parts in step 1 and step 2, respectively. For the construction of (1) an $\mid \widetilde{S_{11} \mid}$ measurement is required. Whereas for (2), both an $\left|\widetilde{S_{11}}\right|$ and $e_{c d}$ measurement are required. The measurement procedures are described in Section IV. Next, the SBO technique that minimizes the cost function is outlined briefly in Section II-C.

\section{Surrogate Based Optimization}

The optimization algorithm used here is the Efficient Global Optimization (EGO) formulation as proposed in [34]. This surrogate based optimization (SBO) technique relies on the construction of kriging surrogate models, which provide an approximate mapping between the optimization variables and the cost function based on well-chosen sample points. First, an

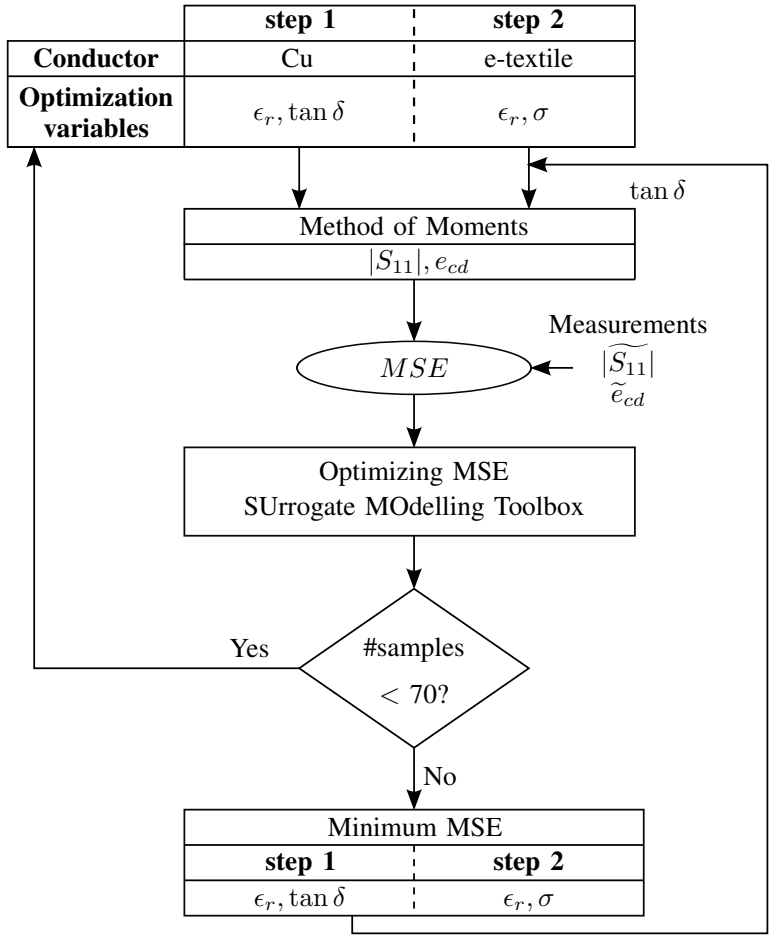

Fig. 2. Schematic representation of the characterization process.

initial kriging model is constructed from a set of sample points determined through a maximum Latin Hypercube Design and the four corner points defined by the upper and lower bounds of the optimization range of the constitutive parameters. In SBO, this global surrogate model provides extra information and helps to search for a global optimum, hence avoiding potential local optima. Subsequently, an adaptive sampling strategy is used for determining the next sample to evaluate and hence update the surrogate model.

Since the focus is on optimization a global accurate kriging model is not required and only a locally accurate kriging model around the optimum is of interest. Therefore EGO uses the expected improvement (EI) criteria as adaptive sampling strategy. These infill criteria yield a compromise between a local search around the global optimum (minimum) and a global search where a high uncertainty of the kriging model is encountered [22]. For this, two auxiliary functions are used for determining the next sample to evaluate. The first one describes the probability of improvement, i.e. the probability of finding a better minimum cost function value over the current minimum function value, whereas the second one quantifies how large this improvement will be. These auxiliary functions require a complete normal distribution in each point, i.e. expected value (=prediction) and variance (=prediction error), of the surrogate model which is provided by the kriging surrogate model through the kriging variance formulation describing the kriging model uncertainty in each point. Note that in the evaluated sample points, the variance is zero.

The iterative procedure of optimizing EI, evaluating the next sample, and hence updating the kriging model is repeated until a stopping criteria is fulfilled: Intuitively, one can stop the 
optimization process when the maximal expected improvement value found, is less than a certain threshold, i.e., when the expected gain of evaluating a new sample over the current minimum cost function value is minimal, no more effort should be made. This threshold can be set by the domain expert as it relates to the minimum achievable cost function value. Unfortunately, the expected improvement depends on the statistical information provided by the kriging variance formulation, and it is known that the prediction variance of Kriging is actually an underestimation leading to a too early termination of the optimization process [35]. Therefore, in this work it is chosen to make use of a sample budget as a stopping criterion. Only a limited number of samples is allocated to the optimization process. In Section III it is experimentally validated that the number of samples can be limited to 70 .

In addition, kriging is by default an interpolation technique. However, since the measurements are prone to errors, the cost functions will be noisy. Therefore, the kriging model was adapted to operate as a regression technique [22]. After the optimization ends, the solution of the characterization process is given by the electromagnetic properties that correspond to the minimum of (1) or (2). In step $1, \epsilon_{r}$ and $\tan \delta$ of the substrate are the outcome of the characterization process. Whereas in step 2, a corrected $\epsilon_{r}$ that includes the effect of the electro-textile and the conductivity $\sigma$ of the e-textile are the results of the characterization process.

\section{Characterization OF THE RO4350B High FREQUENCY LAMINATE}

To validate the accuracy of the proposed optimization procedure, the characterization process was applied to the high frequency laminate RO4350B with the following properties: $h=1.524 \mathrm{~mm}, \epsilon_{r}=3.66, \tan \delta=0.0031$ [36]. The dimensions of the inset-fed patch antenna with a $f_{r}=2.985 \mathrm{GHz}$ are given in Table II. The antenna's reflection coefficient was measured in the frequency range from $2.5-3.5 \mathrm{GHz}$ and the measured efficiency was found to be $e_{c d, f_{r, m}}=70.78 \%$. Version 6.2 of the SUrrogate Modeling (SUMO) Toolbox is used [37]. The optimization bounds are set to $\epsilon_{r}=[3.24 .2]$ and $\tan \delta=\left[\begin{array}{ll}0.0001 & 0.025\end{array}\right]$. The sample budget was limited to 200 .

1) Evaluation of the Optimization Process: An evolution plot of the minimum error function value versus the number of samples for the optimization process relying on (1) and (2) is given in Fig. 3. Starting from the initial set of 24 samples, the EI function starts exploring the design space. By applying $M S E_{1}$ and $M S E_{2}$, the global optimum is quickly located after approximately 57 and 45 samples, respectively. Still, the EI criteria explores other parts of the design space, enhancing the global accuracy of the kriging model (flat parts). For $M S E_{1}$, the region near the global optimum is further explored resulting in a final optimum at sample 85 , whereas for $M S E_{2}$ no further improvement is found. The sampling continues until the sample budget of 200 samples is reached. The optimum substrate properties obtained from (1) and (2) are $\left(\epsilon_{r}=3.7617, \tan \delta=0.0071\right)$ and $\left(\epsilon_{r}=3.7594\right.$, $\tan \delta=0.0048)$, respectively. Compared to the RO4350B substrate properties specified by the manufacturer, a high

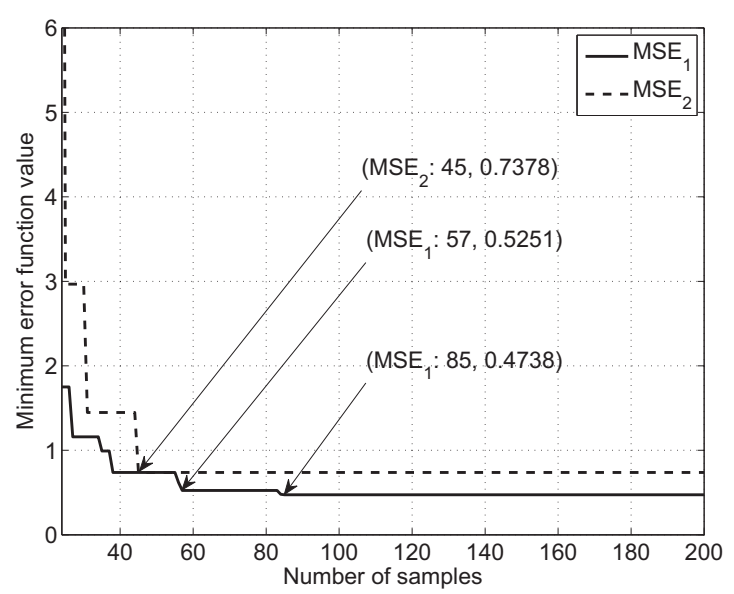

Fig. 3. Comparison in progress of the minimum error function value vs. number of samples between error function $M S E_{1}$ and $M S E_{2}$ applied to the RO4350B reference antenna.

accuracy is achieved for both error functions. For $M S E_{1}$, the found substrate parameters at sample 57 are $\left(\epsilon_{r}=\right.$ $3.7594, \tan \delta=0.0063)$. Additional sampling did not greatly improve the outcome of the characterization process. From these observations we can conclude that a sample budget of 70 samples is adequate and this for limiting the overall time of the characterization process. The average sample time equals $37 \mathrm{~s}$ on an Intel $\mathbb{R}$ Core $^{\mathrm{TM}} 2$ Quad $2.83 \mathrm{GHz}$ CPU, 16 GB RAM system. The final kriging models, resulting from the optimization processes applied to the reference antenna using $M S E_{1}$ and $M S E_{2}$ are depicted in Fig. 4 and Fig. 5, respectively. The kriging model constructed from (1) exhibits the shape of a valley, since $M S E_{1}$ is more affected by a change in $\epsilon_{r}$ than by a change in $\tan \delta$ of the substrate. The kriging model constructed from (2) exhibits a clear optimum thanks to the fact that $\tan \delta$ has a large influence on antenna radiation efficiency. This minimizes the risk of encountering multiple solutions in the vicinity of the global optimum which can be attributed to the finite accuracy of the simulation model combined with measurement errors. However if these exist, after a large amount of time, the EI criteria would find all these solutions. Since the sample budget is limited and the EI infill method still tries to enhance the accuracy of the global kriging model, the probability of encountering multiple solutions nearby the global optimum is relatively small. A comparison between measured and simulated $\left|S_{11}\right|$ using the optimal substrate parameters is illustrated in Fig. 6. Measured and simulated reflection coefficients are in excellent agreement, showing us the outstanding performance of the surrogate-based optimization approach to optimize the error function in a relatively few number of function evaluations.

\section{Characterization OF THE FABRIC AND FoAM MATERIALS}

\section{A. Substrate Materials}

Six different materials, exhibiting different sensitivities to moisture, were investigated. The different substrate materials can be subdivided into two groups according to their moisture 
TABLE I

DESCRIPTION ANTENNA SUBSTRATES

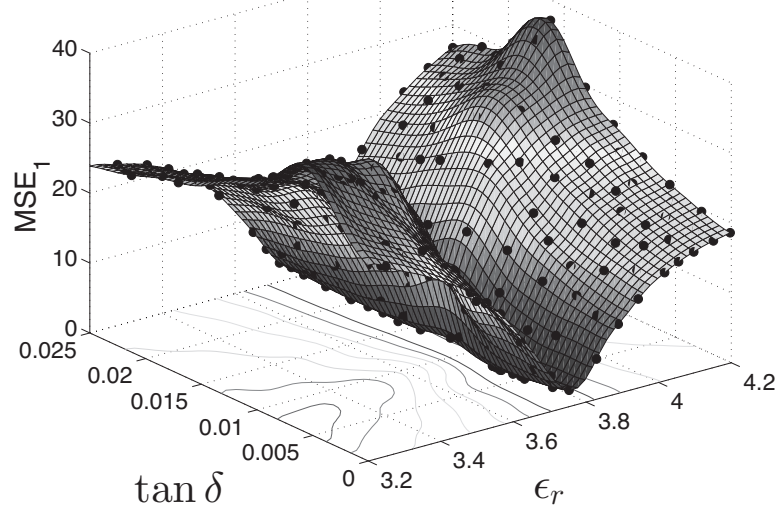

Fig. 4. Final kriging model based on $M S E_{1}$

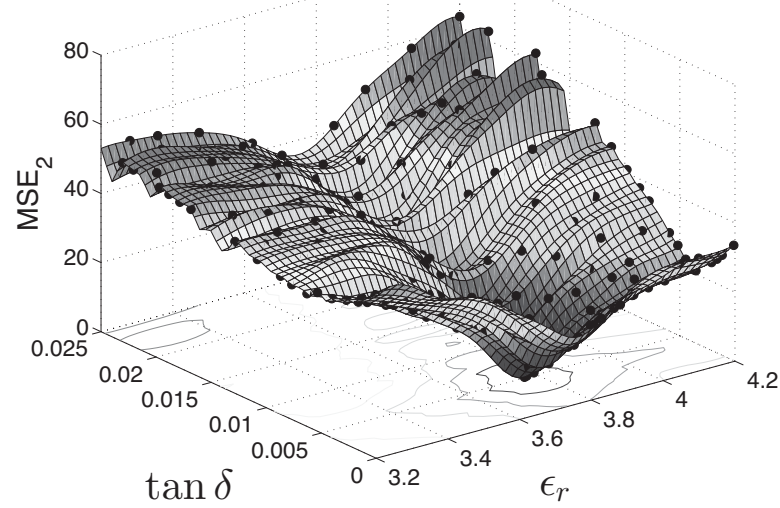

Fig. 5. Final kriging model based on $M S E_{2}$



Fig. 6. Comparison between measured and simulated $\left|S_{11}\right|$ obtained from optimal solutions relying on $M S E_{1}$ and $M S E_{2}$.

\begin{tabular}{|c|c|c|c|}
\hline & antenna substrate composition & $\begin{array}{c}h \\
{[\mathrm{~mm}]}\end{array}$ & $\begin{array}{l}M R \\
{[\%]} \\
\end{array}$ \\
\hline fabric 1 & $\begin{array}{l}4 \text { layers, plain weave: } \\
\text { cotton yarn (weft), linen yarn (warp) }\end{array}$ & 2.4 & $\overline{77.5}$ \\
\hline fabric 2 & $\begin{array}{l}4 \text { layers, twill weave: } \\
100 \% \text { cotton fibres }\end{array}$ & 2.3 & 6.9 \\
\hline fabric 3 & $\begin{array}{l}4 \text { layers, twill weave: } 50 \% \text { polyamide fibres }+ \\
+50 \% \text { cotton fibres }\end{array}$ & 1.63 & 5.3 \\
\hline fabric 4 & $\begin{array}{l}4 \text { layers, twill weave: } 98 \% \text { polyaramide fibres }+ \\
2 \% \text { antistatic carbon fibres }\end{array}$ & 1.67 & 4.9 \\
\hline foam & polyurethane & 3.60 & 1.7 \\
\hline fabric 5 & $\begin{array}{l}\text { non-woven synthetic fleece fabric: } \\
\text { polyethylenteraftalate (PET) }\end{array}$ & 2.20 & 0.2 \\
\hline flectron & $\begin{array}{l}\text { e-textile: } \\
\text { copper-coated nylon fabric }\end{array}$ & $\mathrm{t}=80 \mu \mathrm{m}$ & 0.5 \\
\hline
\end{tabular}

regain $M R$ [14], defined by

$$
M R=\frac{m_{\text {cond }}-m_{d r y}}{m_{d r y}} \times 100[\%]
$$

with $m_{\text {cond }}$ being the weight of the material at equilibrium after conditioning in the climate chamber and $m_{d r y}$ being the mass of the material in dry state. An equilibrium is reached when no further transport of water between the material and its surrounding environment occurs. In this paper, we use the $M R$ as defined according to the $I S O$ standard 6417 (1-4), implying a conditioned state after 24 hours of passive conditioning at $20^{\circ} \mathrm{C}$ and a relative humidity level of $65 \%$. The dry state was reached after drying the material for five hours in an oven at $105^{\circ} \mathrm{C}$. The composition, substrate thickness $h$ and $M R$ for all substrate materials considered are given in Table I. The first group exhibits a high $M R$ and includes four antenna substrates in which each substrate is constructed using an assembly of four woven textile layers in order to provide sufficient thickness for antenna applications. All layers are glued by means of an adhesive sheet. The second group consists of two synthetic substrates, being a foam material and a fleece fabric. Concerning the measured moisture regains, we conclude that the substrates based on the woven fabrics are more hydrophilic compared to the non-woven fabric and foam substrate. This hydrophilic property of the woven natural and synthetic fabrics results from the open weave structure of the fabrics. Also note that natural fibres are more hydrophilic than synthetic fibres. The foam and fleece fabric exhibit the lowest $M R$ and are therefore the most water repellent materials in this study. The foam materials are open cell structures and tend to absorb some moisture as can be seen from the $M R$. Also the moisture regain of the e-textile was measured and is listed in Table I. The e-textile used here is a copper plated polyester, plainwoven fabric with a tarnish resistant coating. The thickness $t$ of the e-textile is $80 \mu \mathrm{m}$. This tarnish resistant coating avoids oxidation of the e-textile. A DC surface resistivity, $R_{s}=0.05 \Omega / s q$ is specified by the manufacturers [38]. 
TABLE II

DIMENSIONS OF THE COPPER AND ELECTROTEXTILE BASED FABRIC ANTENNAS IN [MM].

\begin{tabular}{c|c|c|c|c|c|c|c}
\hline \hline copper & $L$ & $W$ & $x_{f}$ & $L_{f}$ & $s_{i}$ & $w_{f}$ & ground plane size \\
\hline \hline fabric 1 & 43 & 55 & 8 & 28 & 2 & 9 & $80 \times 80$ \\
\hline fabric 2 & 43.5 & 55 & 8 & 32 & 2 & 8 & $85 \times 85$ \\
\hline fabric 3 & 43 & 55 & 8 & 28 & 2 & 6 & $80 \times 90$ \\
\hline fabric 4 & 43 & 55 & 8.5 & 25 & 2 & 6 & $75 \times 80$ \\
\hline foam & 53 & 70 & 13 & 32 & 3 & 16 & $90 \times 100$ \\
\hline fabric 5 & 55 & 69 & 13 & 32 & 3 & 9 & $95 \times 85$ \\
\hline \hline flectron & $L$ & $W$ & $x_{f}$ & $L_{f}$ & $s_{i}$ & $w_{f}$ & ground plane size \\
\hline \hline fabric 1 & 43 & 55 & 7 & 26 & 2 & 9 & $75 \times 80$ \\
\hline fabric 2 & 43 & 55 & 7 & 27 & 2 & 8 & $80 \times 90$ \\
\hline fabric 3 & 42 & 55 & 5 & 25 & 2 & 6 & $85 \times 100$ \\
\hline fabric 4 & 44 & 55 & 7 & 24 & 2 & 6 & $80 \times 90$ \\
\hline foam & 55 & 70 & 11 & 36 & 3 & 16 & $100 \times 105$ \\
\hline fabric 5 & 55 & 70 & 13 & 33 & 3 & 10 & $95 \times 90$ \\
\hline \hline RO4350B & 25.5 & 32.3 & 7.7 & 17.7 & 1 & 4.2 & $57 \times 67$ \\
\hline \hline
\end{tabular}

\section{B. Antenna Design}

For each textile substrate, two antenna prototypes, one etextile based and one copper foil based antenna were constructed. The antennas were designed to operate in the vicinity of $2.45 \mathrm{GHz}$. The conductive layers were glued onto the substrates using an adhesive sheet. The ground plane size of the antennas was chosen large enough so that the effect of a finite size ground plane did not affect the resonance frequency, i.e. keeping at least a $\lambda / 20$ distance between the patch edge and the substrate edge [39]. The resulting dimensions of the inset fed copper based and e-textile based antennas are given in Table II.

\section{Measurement Setup}

1) Climate Chamber Setup: The twelve antenna prototypes were placed in a climate chamber (WK 350 from Weiss Technik) and conditioned at a specific relative humidity level at $23^{\circ} \mathrm{C}$ and this for 24 hours, assuming that an equilibrium state was reached between the moisture absorbed by the material and the moisture present in the climate chamber. The relative humidity levels that were tested are 10, 20, 30, 40, 50, 60, 70,80 and $90 \%$, resulting in a total of nine days required for executing the measurement campaign.

2) Reflection Coefficient Measurements: After 24 hours of conditioning at one specified $R H$ level each antenna was removed from the climate chamber to immediately perform a free-space reflection coefficient measurement in the frequency range from $2 \mathrm{GHz}$ to $3 \mathrm{GHz}$. Here, the 8714 ET Network Analyzer from Agilent Technologies was utilized and the number of frequency points in the measured range was set to 801 , yielding a resonance frequency measurement accuracy of $1.25 \mathrm{MHz}$. The measurements were executed as quickly as possible since removing the antenna from the climate chamber imposes a disequilibrium between the moisture absorbed in the antenna and the unknown moisture present in the prevailing atmosphere, resulting in a transfer of moisture between antenna and its surrounding atmosphere. However, the level of

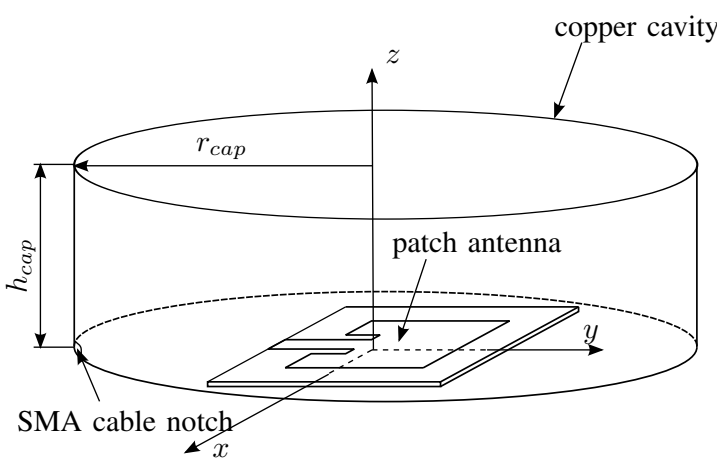

Fig. 7. Schematic representation of the planar textile antenna encapsulated by the copper cavity.

humidity inside the substrate is assumed to remain unchanged for measurements that are performed sufficiently fast. After each measurement, the antenna is placed back inside the climate chamber. After the reflection coefficient measurements of all twelve prototypes, the antennas were conditioned to the next relative humidity level and this for 24 hours. This process was repeated until all reflection coefficients of the twelve prototypes at nine different $R H$ levels were measured.

3) Antenna Radiation Efficiency Measurements: For the efficiency measurements, the radiation pattern method is unfeasible since the measurement itself is too time consuming in order to ensure a constant level of humidity inside the substrate during the measurement. Therefore, we chose to use the Generalized Wheeler cap method as described in [40]. This method calculates the radiation efficiency of antennas by measuring the antenna input impedance for two different conditions. The first one is the free-space measurement whereas the second one is performed by placing the antenna inside a closed metallic cavity. Hence, each free-space reflection coefficient measurement as described in Section IV-C was accompanied by a reflection coefficient measurement with the antenna positioned inside a copper cavity as shown in Fig. 7. The size of the cap was designed in order not to disturb the near-field distribution of the antenna. A closed circular copper cavity was used with a radius $r_{c a p}$ of $86 \mathrm{~mm}$ and a height $h_{\text {cap }}$ of $50 \mathrm{~mm}$. The excited eigenmodes of the cavity below $3 \mathrm{GHz}$ are the $T M_{010}^{z}, T M_{110}^{z}, T M_{210}^{z}$ and $T E_{111}^{z}$ modes, which occur at $1.33 \mathrm{GHz}, 2.13 \mathrm{GHz}, 2.85 \mathrm{GHz}$ and $3 \mathrm{GHz}$, respectively. A schematic overview of the antenna inside the metallic cavity is depicted in Fig. 7.

\section{Results and Validation of the Textile SUBSTRATES}

The first step of the characterization process is applied to the copper foil based antennas in order to extract $\epsilon_{r}$ and $\tan \delta$ of the foam and fabric antenna substrates described in Table I. First, the results obtained through the use of error function $M S E_{1}$ are discussed. This to highlight the need for the improved error function $M S E_{2}$. Next, we describe the results obtained using error function $M S E_{2}$. In order not to overload this section, only the results of 4 materials are discussed and presented. 


\section{A. Surrogate Modeling Optimization Setup}

Concerning the characterization in step 1 , for the copperbased antennas, constructed from fabric 5 and the foam material - which have an estimated permittivity close to one and a small $M R$ - the bounds are set to $\epsilon_{r}=\left[\begin{array}{ll}1 & 1.5\end{array}\right]$ and $\tan \delta=[0.00010 .05]$. For the substrate materials, exhibiting high $M R$ with an estimated $\epsilon_{r} \approx 1.8$, the bounds are chosen $\epsilon_{r}=\left[\begin{array}{ll}1.5 & 2.6\end{array}\right]$ and $\tan \delta=\left[\begin{array}{ll}0.005 & 0.15\end{array}\right]$. For the characterization process applied to the e-textile based antennas, i.e. step two, in which loss tangent extracted from step one is reused, the permittivity optimization range was taken identical to step one. In this second step, the bounds of the effective conductivity are $\sigma=\left[5.0 \times 10^{3} \mathrm{~S} / \mathrm{m} 5.0 \times 10^{6} \mathrm{~S} / \mathrm{m}\right]$.

\section{B. Step 1 of the Characterization Process.}

The extracted $\epsilon_{r}$ and $\tan \delta$ at $R H=40 \%$ of the substrates fabric 1, fabric 4, fabric 5 and the foam substrate as well as the optimum error function value $M S E_{1, o p t}$, are given in Table III. The absolute error between simulated and measured resonance frequencies $\left|\Delta f_{r}\right|=\left|f_{r, s}-f_{r, m}\right|$ and the absolute radiation efficiency errors $\left|\Delta e_{c d, f_{r}}\right|=\left|e_{c d, f_{r, s}}-e_{c d, f_{r, m}}\right|$ are also listed in this Table. The results illustrated in Table III demonstrate a very good estimation of the extracted permittivity for all materials since the resonance frequency error $\left|\Delta f_{r}\right|$ is very small, taking into account a measurement resolution of $1.25 \mathrm{MHz}$. Observing the optimal error function values in Table III, the results for fabric 4 exhibit the highest accuracy, whereas the results obtained for the foam substrate yields the largest error function value and thus the lowest accuracy. However, a minimal error function value does not automatically imply the highest accuracy of characterization due to uncertainties in the simulation model, as described in Section II-B, combined with measurement uncertainties. This is observed when comparing the results from fabric 1 and fabric 5. Fabric 5 exhibits a smaller $M S E_{1, o p t}$ compared to fabric 1 but has a larger $\left|\Delta e_{c d, f_{r}}\right|$. Assuming an exact knowledge of the antenna patch length $L$ and a $\left|\Delta f_{r}\right|=$ $1.3 \mathrm{MHz}$ for fabric 1 and fabric 5 implies the same error in extracted $\epsilon_{r}$ for both substrates. From these two observations, we conclude that the estimated $\tan \delta$ of fabric 5 exhibits the largest error.

TABLE III

OPTIMAL MATERIAL PARAMETERS EXTRACTED FROM MEASUREMENTS AT $R H=40 \%$

\begin{tabular}{c|c|c|c|c|c|c}
\hline \hline$R H=40 \%$ & $\begin{array}{c}h \\
{[\mathrm{~mm}]}\end{array}$ & $\epsilon_{r}$ & $\tan \delta$ & $M S E_{1, \text { opt }}$ & $\begin{array}{c}\left|\Delta f_{r}\right| \\
{[\mathrm{MHz}]}\end{array}$ & $\begin{array}{c}\left|\Delta e_{c d, f_{r}}\right| \\
{[\%]}\end{array}$ \\
\hline \hline fabric 1 & 2.40 & 1.767 & 0.032 & 0.220 & 1.3 & 6.4 \\
\hline fabric 4 & 1.67 & 1.849 & 0.015 & 0.085 & 2.5 & 2.1 \\
\hline foam & 3.60 & 1.237 & 0.021 & 0.421 & 1.3 & 0.4 \\
\hline fabric 5 & 3.80 & 1.173 & 0.006 & 0.100 & 1.3 & 9.9 \\
\hline \hline
\end{tabular}

Fig. 8 and Fig. 9 depict the extracted permittivities and loss tangents for all substrates as a function of relative humidity, respectively. Fabric 1 and fabric 4 exhibit a clear increase in permittivity and loss tangent for increasing relative humidity levels due to their high $M R$. Furthermore, from the $\epsilon_{r}$ 's and $\tan \delta$ 's as a function of $R H$ it is clearly visible that fabric 1 has higher $M R$ compared to the fabric 4 substrate since the increase in $\epsilon_{r}$ and $\tan \delta$ is more pronounced for increasing $R H$ levels. As reported in [14], for these fabrics, a quadratic behavior of the permittivity as a function of $R H$ is observed. For the foam and fabric 5 substrate it is not possible to compare their $M R$ by observing the behavior of $\epsilon_{r}$ and $\tan \delta$ as a function of $R H$ and this due to geometrical uncertainties combined with measurement errors and the small $M R$ value. In Fig. 9 we see that $\tan \delta$ as a function of $R H$ exhibits some discrepancies as the results are not monotonically increasing for increasing $R H$. In order to further verify the obtained results as a function of relative humidity, a comparison between measured and simulated antenna efficiencies and all $\left|\Delta f_{r}\right|$ 's are depicted in Fig. 10 and Fig. 11, respectively. The simulated efficiencies and resonance frequencies are calculated using the electromagnetic properties depicted in Fig. 8 and Fig. 9. From the resonance frequency errors we conclude that the extracted permittivities are very accurate since most resonance frequency errors remain below $4 \mathrm{MHz}$. By comparing simulated and measured radiation efficiencies, we conclude that the extracted loss tangent results are accurate for fabric 1, fabric 4 and the foam substrate, keeping in mind that the measured antenna radiation efficiencies also exhibit discrepancies due to the measurement accuracy. For the fabric 5 substrate, it is observed that all extracted $\tan \delta$ 's are overestimated since $e_{c d, f_{r, s}}<\widetilde{e_{c d}, f_{r, m}}$ and this for all $R H$ levels. Note that $e_{c d}$ depends both on $\tan \delta$ and $\epsilon_{r}$, and since extracted $\epsilon_{r}$ 's show a good accuracy, the discrepancies between simulated and measured $e_{c d}$ allows verifying the accuracy of the estimated $\tan \delta$ 's.

From the results of the characterization process based on the error function (1) applied to the copper foil based antennas, we conclude that a very good accuracy is obtained in extracted complex permittivities of the fabric 1 and fabric 4 substrate. For the two remaining substrates, the estimated loss tangents are less accurate. As seen in the kriging surrogate model given in Fig. 4, the error function's dependency on loss tangent is poor. This, together with the simplifications made in the simulation model, yields larger errors in the characterization process. More in particular, for thicker substrates connector mounting is harder to realize, introducing additional losses that are not accounted for in the simulation model. If these thicker substrates exhibit low $\tan \delta$, the additional losses caused by the low-quality connector mounting will dominate the losses that are captured in the measured reflection coefficient of the antenna.

1) Results Based on Error Function $M S E_{2}$ : For low-loss thick substrates, the use of error function $M S E_{1}$ in step 1 yields a large error in estimated $\tan \delta$. However, the extracted $\tan \delta$ from step 1 is used in step 2 of the characterization process. Therefore, we must know $\tan \delta$ with a high degree of accuracy in order to obtain a good estimation of the e-textile's effective conductivity. Therefore, $M S E_{2}$ given by (2) was utilized for extracting $\epsilon_{r}$ and $\tan \delta$ in step 1 . Since $M S E_{2}$ includes a comparison between measured and simulated antenna efficiency, the kriging model will exhibit a higher dependency 


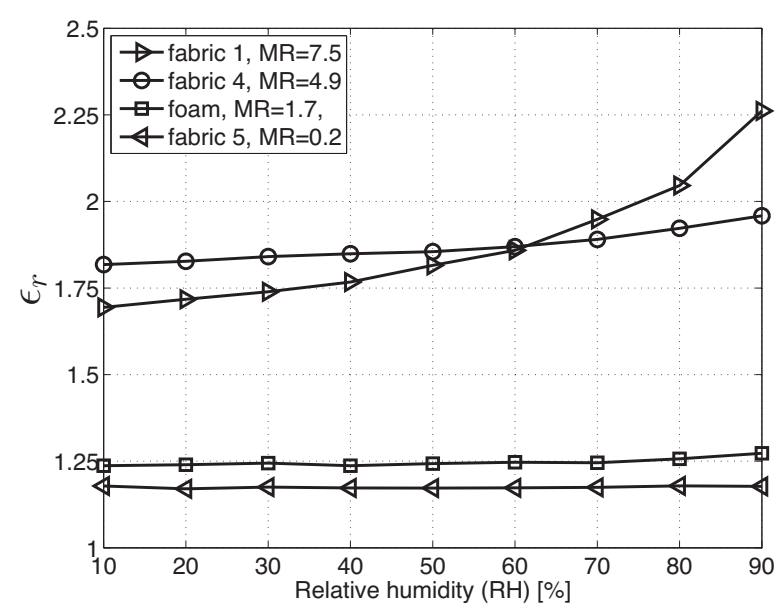

Fig. 8. Estimated permittivities as a function of RH, utilizing error function $M S E_{1}$.

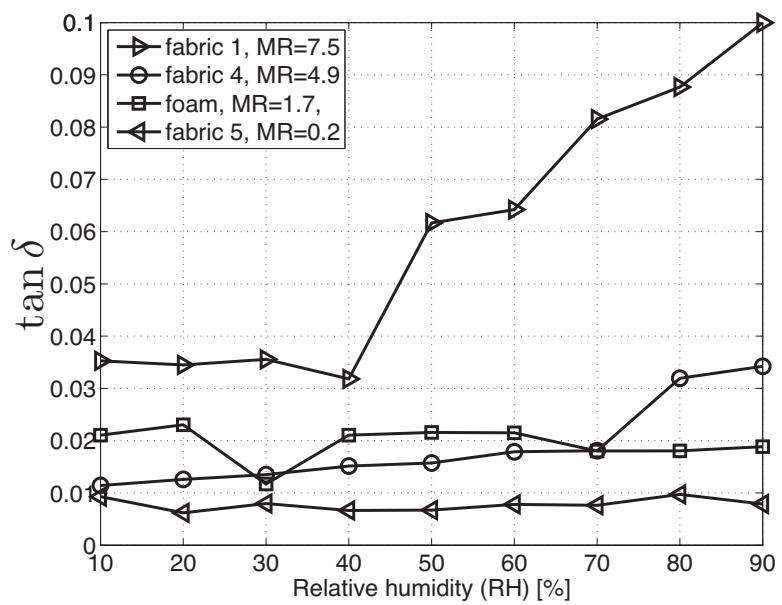

Fig. 9. Estimated loss tangents as a function of RH, utilizing error function $M S E_{1}$.

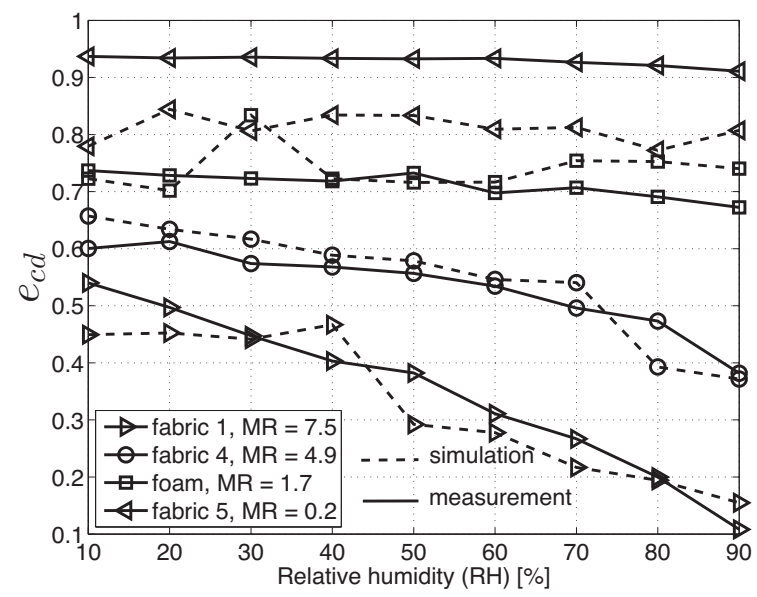

Fig. 10. Comparison between measured and simulated $e_{c d}$ at their respective $f_{r}$. Simulation results based on estimated dielectric properties, utilizing error function $M S E_{1}$.

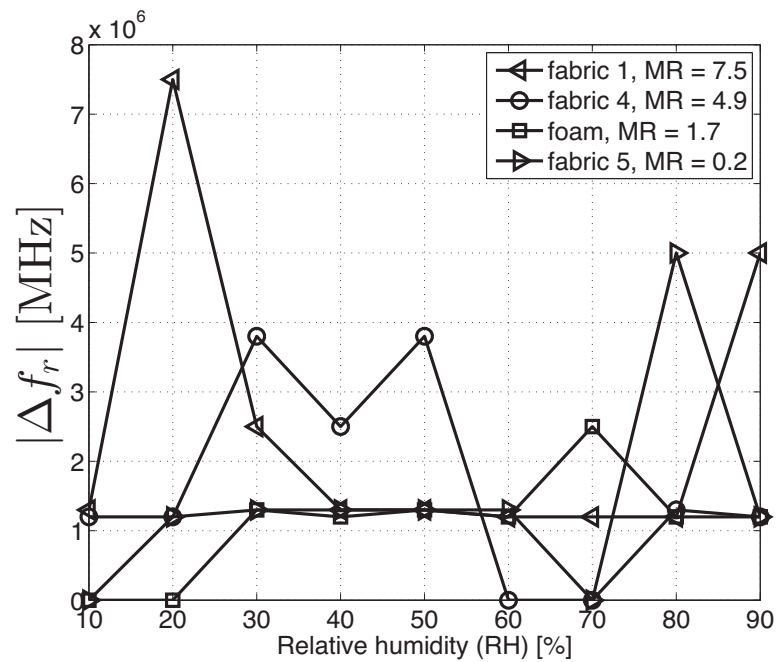

Fig. 11. Resonance frequency errors, based on estimated dielectric properties utilizing error function $M S E_{1}$.

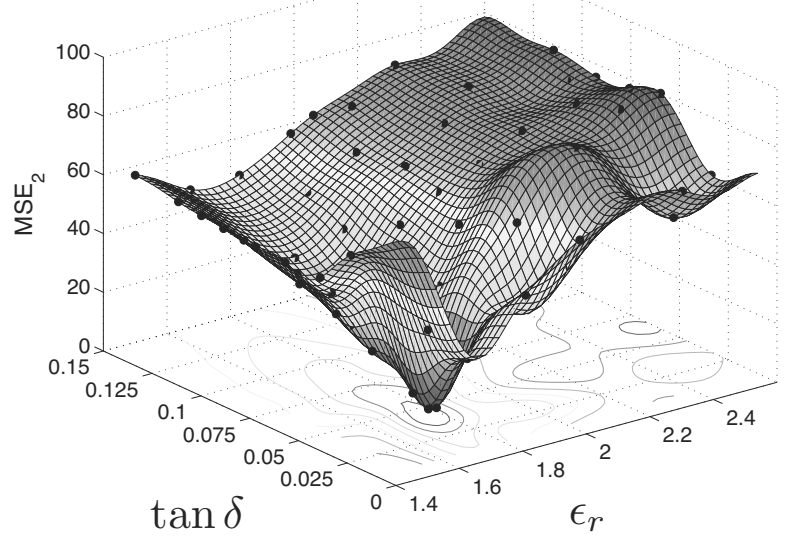

Fig. 12. Final kriging model based on error function $M S E_{2}$ in step 1 copper based fabric 1 antenna, $\mathrm{RH}=40 \%$

on loss tangent. As outlined in Section II, the weighting factors $a_{1}$ and $a_{2}$ in (2) are chosen 1 and 100, respectively.

The final kriging model, resulting from the optimization applied to the copper-based antenna relying on fabric 1 at $R H=40 \%$ is depicted in Fig. 12. The valley shape is no longer present in the kriging model and the optimum substrate's $\epsilon_{r}$ and $\tan \delta$ is clearly visible. In order not to overload the paper, only extracted $\tan \delta$ 's as a function of $R H$ are given in Fig. 13. A clear relation between material $M R$ and the slope of the $\tan \delta$ curve as a function of $R H$ is observed. Even a slightly larger increase in $\tan \delta$ is observed for the foam compared to the fabric 5 material, as expected from their $M R$. Clearly, to characterize loss tangent, utilizing (2) in the characterization process yields more accurate results compared to (1).

\section{Step 2 of the Characterization Process.}

The second step of the characterization process is applied to the e-textile based antennas in order to extract the equivalent $\epsilon_{r}$ of the foam and fabric antenna substrates as well as the 


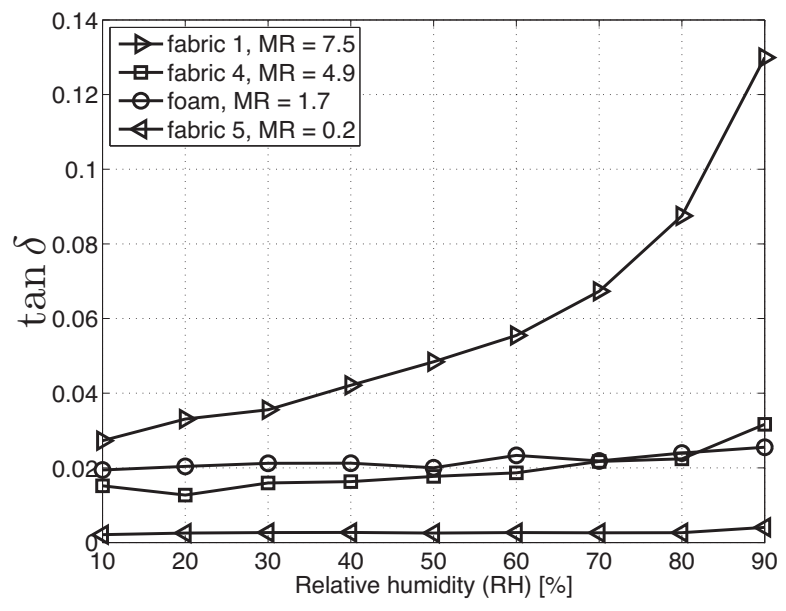

Fig. 13. Estimated loss tangent as a function of $R H$, utilizing error function $M S E_{2}$.

$\sigma$ of the e-textile as described in Table I. Since the error function $M S E_{1}$ was inadequate in step 1 for an accurate characterization of the $\tan \delta$, it was not utilized in step 2 of the characterization process. Therefore only $M S E_{2}$ was used in step 2 of the characterization process.

1) Results Based on Error Function $\mathrm{MSE}_{2}$ : The $\tan \delta$ extracted from step 1 is now used in step 2 of the characterization process and is kept constant for each $R H$ level. Now, $\epsilon_{r}$ and $\sigma$ are optimized in order to obtain a minimum error function value and hence extract the equivalent substrate $\epsilon_{r}$ and $\sigma$ of the e-textile. The final kriging model, resulting from the characterization process applied to the e-textile antenna based on fabric 4 at $R H=30 \%$ is depicted in Fig. 14. Again the shape of a valley is observed in the kriging model. For low conductivity values. i.e. for $\sigma=5.0 \times 10^{3}-0.5 \times 10^{6} \mathrm{~S} / \mathrm{m}$, the kriging model is more sensitive to changes in $\sigma$ compared to the region where $\sigma>0.5 \times 10^{6} \mathrm{~S} / \mathrm{m}$. This due to the fact that $e_{c d}$ drops rapidly when conductivity is relatively poor. Hence, for relatively large conductivity of the e-textile, the global optimum is less pronounced and the resulting optimum will be more affected by measurement errors and simulation model uncertainty. The obtained $\epsilon_{r}$ 's as a function of $R H$ of all substrates are depicted in Fig. 15. The extracted equivalent conductivity of the e-textile and the surface resistivity $R_{s}$, calculated by $R_{s}=\sqrt{\pi f_{r, s} \mu_{0} / \sigma}$ are depicted in Fig. 16. As expected, no decreasing conductivity is observed for increased $R H$ levels thanks to the tarnish resistant finish of the e-textile. The extracted conductivity lies in the range of $10^{5}-10^{6} \mathrm{~S} / \mathrm{m}$ yielding a corresponding average surface resistivity of about $0.2 \Omega / s q$. A comparison between measured and simulated antenna efficiencies $e_{c d}$ at their respective resonance frequencies as a function of $R H$ for all materials is shown in Fig. 17. Since measured efficiency is used in construction of the error function $M S E_{2}$, the comparison cannot be used to validate the results. They only show us how well the surrogate based optimization finds a fit between measured and simulated $e_{c d}$. The absolute errors in resonance frequency depicted in Fig. 18 demonstrate accurate results for $\epsilon_{r}$. However, they are slightly larger compared to the $\Delta f_{r}$ 's

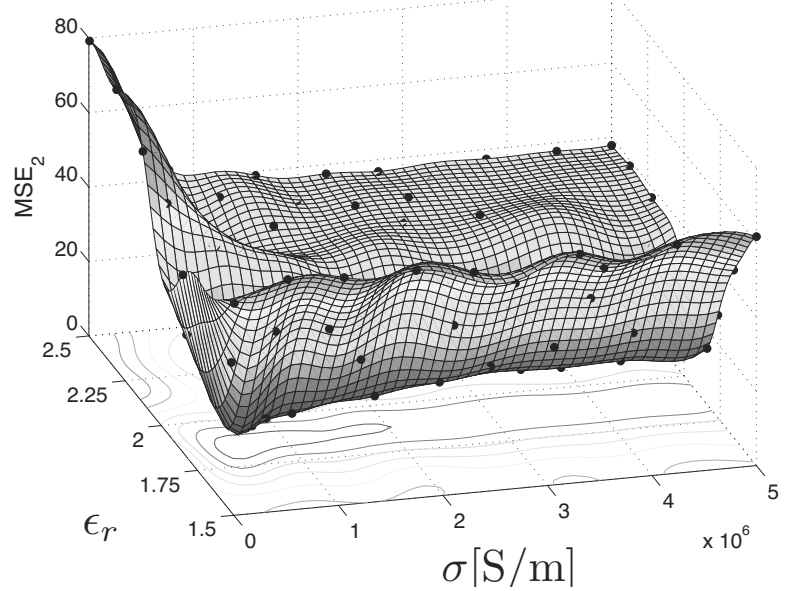

Fig. 14. Final kriging model step 2, e-textile based fabric 4 antenna, RH=30\%

depicted in Fig. 11. This due to the fact that both efficiency and reflection coefficient data are used in the error function. Hence, the choice of the weighting coefficients $a_{1}$ and $a_{2}$ influences the outcome of the characterization process since measurement errors and simplifications made in the simulation model are responsible for different optimum values in the first and second part of the error function $M S E_{2}$. Ideally, when measurement errors are small and a highly accurate simulation model is available, then a fit between measured and simulated $\left|S_{11}\right|$ automatically yields an optimal resemblance between measured and simulated $e_{c d}$. Furthermore, any uncertainty in extracted $\tan \delta$ from step 1 contributes to the uncertainty of the simulation model in step 2 of the characterization process.

In general it is shown that error function $M S E_{1}$ is capable of extracting the substrate's $\epsilon_{r}$ with a very good accuracy. Determining complex permittivity requires the use of error function $M S E_{2}$, as a change in loss tangent has a larger influence on antenna efficiency than on antenna bandwidth, as captured in the reflection coefficient of the antenna. A comparison between the kriging models in Fig. 12 and Fig. 14 shows a more pronounced optimum in the first kriging model constructed from the optimization process applied to a copper based antenna. From this we conclude that error function $M S E_{2}$ is more sensitive to changes in loss tangent of the substrate than to variations in $\sigma$ of the e-textile. Hence, geometrical uncertainties or simulation model inaccuracies will have a larger influence on the outcome of step 2 in the characterization process compared to step 1 .

\section{Dielectric MiXing Model}

We now apply the Birchak [31] mixing model to estimate the macroscopic complex $\epsilon=\epsilon^{\prime}-j \epsilon$ " of the fabric and foam substrates as a function of relative humidity with a constant temperature. For each $R H$ level, the substrates absorb a specific amount of moisture. This is represented by a volumetric fraction that quantifies the volume of water inside the substrate. The Birchak mixing model estimates the macroscopic complex $\epsilon$ of the heterogenous substrate by relying on the volumetric fractions of the constituents. For $n$ constituents with volumetric fractions $V_{i}$ and respective 


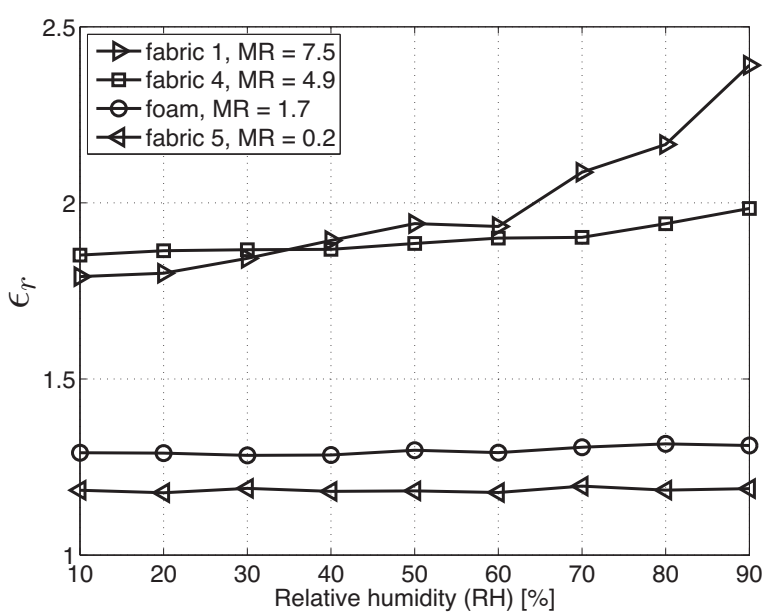

Fig. 15. Step 2: estimated $\epsilon_{r}$ 's vs. $R H$, utilizing error function $M S E_{2}$.
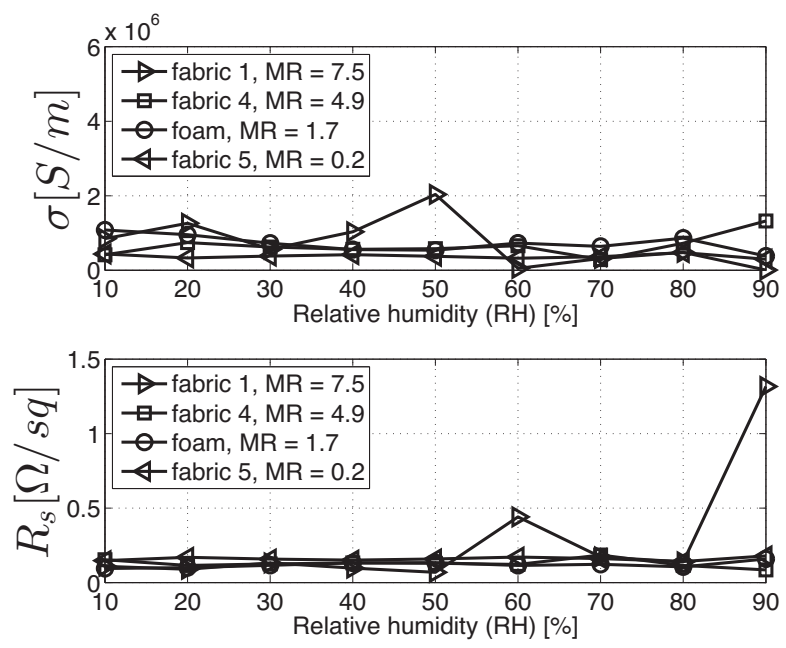

Fig. 16. Step 2: estimated $\sigma$ 's and $R_{s}$ vs. $R H$ of the e-textile, utilizing error function $M S E_{2}$.

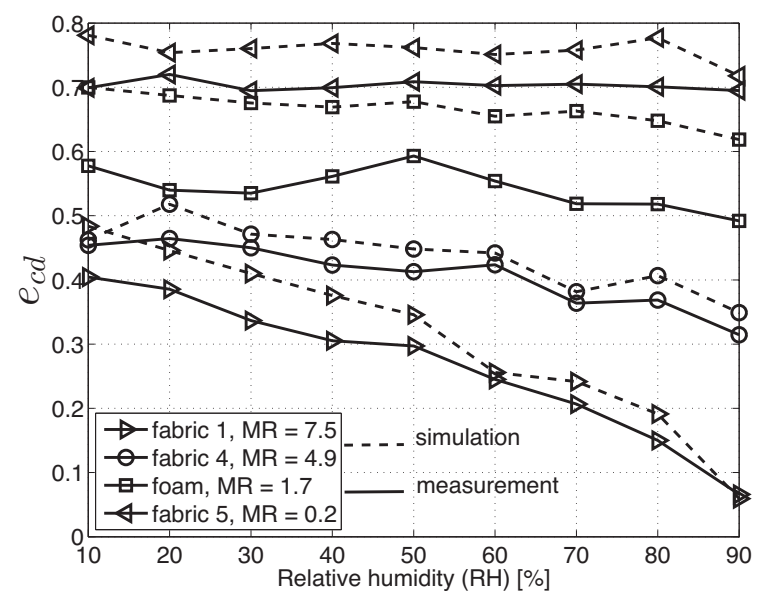

Fig. 17. Comparison between measured and simulated $e_{c d}$. Simulation results based on estimated dielectric properties and conductivity, utilizing error function $M S E_{2}$.

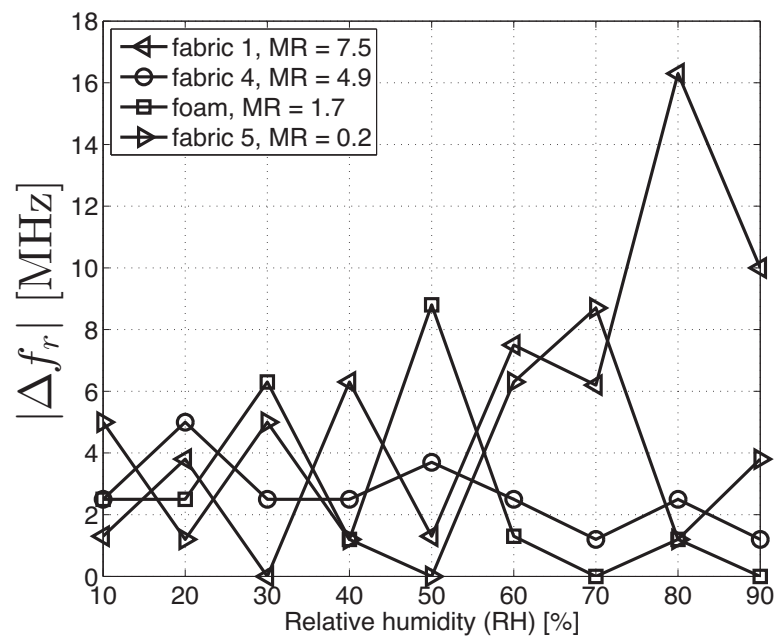

Fig. 18. Resonance frequency errors, based on estimated dielectric properties and conductivity, utilizing error function $M S E_{2}$.

complex $\epsilon_{i}$, the macroscopic permittivity of foam/fabric is given by [31]

$$
\epsilon^{\alpha}=\sum_{i}^{n} V_{i} \epsilon_{i}^{\alpha},
$$

with $\alpha$ being a constant shape factor. For fabric and foam materials, which are three-phase materials consisting of bulk material, air and foam, we have that

$$
\epsilon^{\alpha}=V_{m} \epsilon_{m}^{\alpha}+V_{w} \epsilon_{w}^{\alpha}+V_{a}^{\alpha}
$$

where the subscripts $m, w$ and $a$ refer to the bulk fabric or foam material, water and air, respectively. Since the volumetric fraction of air is not easily determined, we make an approximation by assuming that the bulk fabric material and air form one homogenous substrate with a complex permittivity and volumetric fraction denoted by $\epsilon_{m, a}$ and $V_{m, a}$, respectively. Hence, (5) simplifies to

$$
\epsilon^{\alpha}=V_{m, a} \epsilon_{m, a}^{\alpha}+V_{w} \epsilon_{w} .
$$

In (6), one can easily measure $V_{w}$ at one specific $R H$ level by comparing the dry mass of the substrate with the conditioned mass obtained by conditioning the substrates in the climatic chamber according to the procedure presented in Section IV. Hence, the volumetric fraction of the bulk material/air substrate equals $V_{m, a}=1-V_{w}$. In addition we neglect the small increase in volume of the substrate due to swelling of the fibres when absorbing moisture. It is clear that both simplifications reduce the accuracy of the mixing model at high $R H$-levels and for high $M R$ materials. The complex $\epsilon_{m, a}$ of the dry substrates were determined by means of the characterization procedure applied to the copper based antennas. The value of the complex permittivity $\epsilon_{w}$ of water was calculated according to the Debye dispersion model as presented in [41]. The value of the shape factor $\alpha$ was determined separately for $\epsilon^{\prime}$ and $\epsilon$ " by minimizing the mean squared error between the measured and modeled $\epsilon^{\prime}$ and $\epsilon^{\prime \prime}$. The measured $\epsilon^{\prime}$ and $\epsilon^{\prime \prime}$ are obtained from the characterization process applied to the copper based antennas 


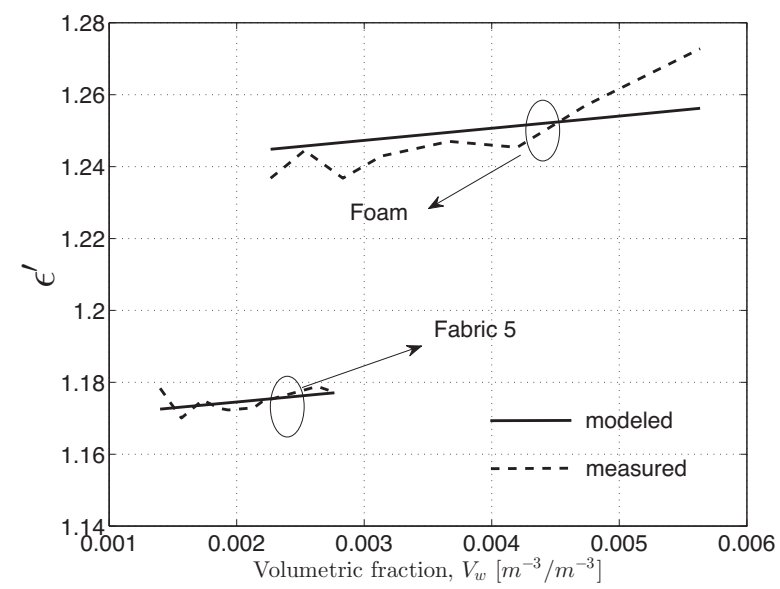

Fig. 19. Comparison between the measured and estimated $\epsilon_{r}$ by relying on the Birchak Mixing model of the substrates exhibiting low $M R$.

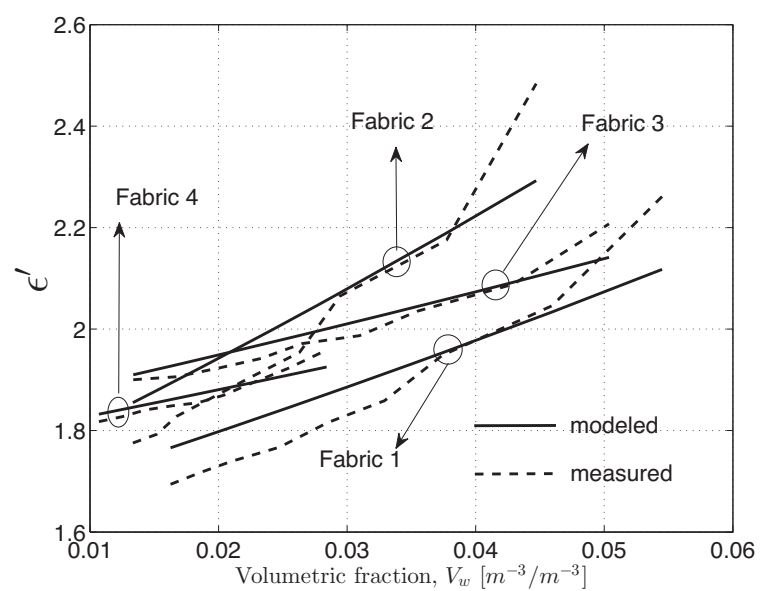

Fig. 20. Comparison between the measured and estimated $\epsilon_{r}$ by relying on the Birchak Mixing model of the substrates exhibiting high $M R$.

using the cost function $M S E_{1}$ and $M S E_{2}$, respectively, as $M S E_{1}$ provides more reliable results for the real part of the complex permittivity whereas $M S E_{2}$ yields more reliable loss tangents of the substrate materials. A comparison between the measured and modeled $\epsilon^{\prime}$ for the substrates exhibiting high MR and low MR is given in Fig. 19 and Fig. 20, respectively. Measured and modeled $\epsilon$ " is given in Fig. 21 and Fig. 22 for the substrate materials exhibiting high $M R$ and low $M R$, respectively. Generally, it is observed that the model overestimates complex permittivity of the substrates for low volumetric fractions of water $V_{w}$ whereas an underestimation is seen at high $V_{w}$. Also, the model exhibits a linear behavior of complex permittivity as a function of $V_{w}$ which is not observed in the measurements of the high $M R$ fabrics 1, 2, 3 and 4 . However the slope of this linear behavior fits the measured data relatively well.

The accuracy of the dielectric mixing model prediction for $\epsilon^{\prime}$ and $\epsilon^{\prime \prime}$ is listed in Table IV and Table V, respectively. The absolute mean error between the modeled and measured real part and imaginary part of the complex permittivity is denoted

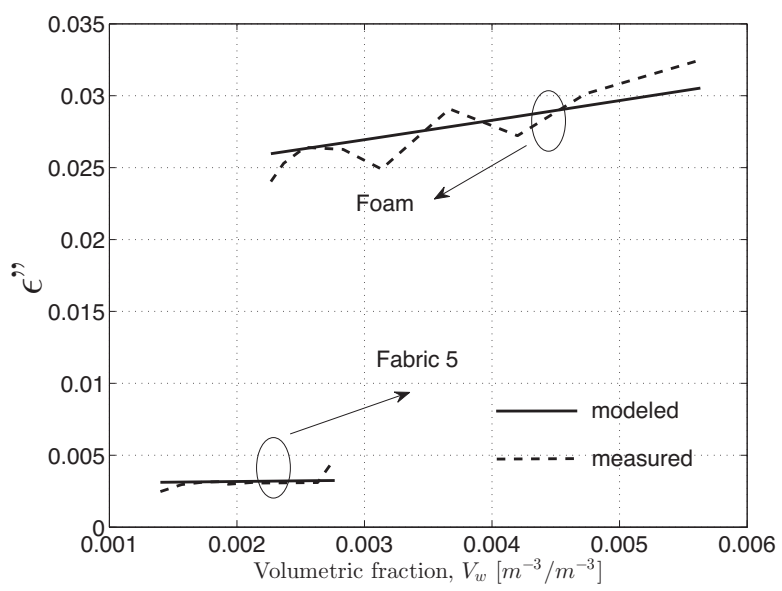

Fig. 21. Comparison between the measured and estimated $\epsilon$ " by relying on the Birchak Mixing model of the substrates exhibiting low $M R$.

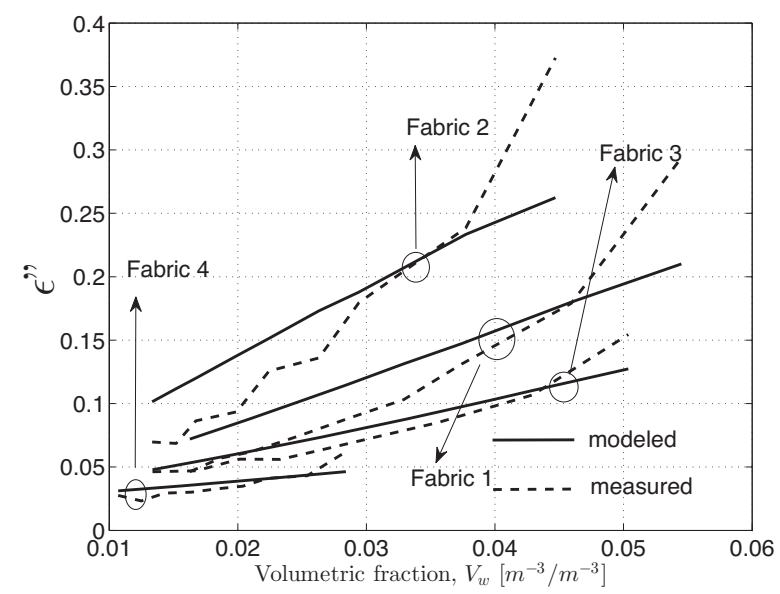

Fig. 22. Comparison between the measured and estimated $\epsilon$ " by relying on the Birchak Mixing model of the substrates exhibiting high $M R$.

by $\overline{\Delta \epsilon^{\prime}}$ and $\overline{\Delta \epsilon^{\prime \prime}}$, and the corresponding standard deviation is denoted by $S D$. In order to compare the accuracy of the predicted $\epsilon^{\prime}$ with the accuracy of the characterization method, we extracted $\epsilon^{\prime}$ for all substrate materials at $R H=40 \%$ and this for a geometrical tolerance of $+/-0.5 \mathrm{~mm}$ in the length of the patch $L$. Since the length of the patch has the largest influence on the resonance frequency of the antenna, an estimation of the characterization procedure's accuracy with respect to the calculation of $\epsilon^{\prime}$ is provided by this approach. The absolute errors between extracted $\epsilon$ for $L-0.5 \mathrm{~mm}$ and $L+0.5 \mathrm{~mm}$ and the nominal extracted $\epsilon^{\prime}$ at $R H=40 \%$ are denoted by $\Delta \epsilon_{L+0.5 \mathrm{~mm}}^{\prime}$ and $\Delta \epsilon_{L-0.5 \mathrm{~mm}}^{\prime}$, respectively and are also given in Table IV. Comparing $\overline{\Delta \epsilon^{\prime}}$ with the estimated accuracy of the characterization procedure shows us that the error of the dielectric mixing model is comparable for the fabric substrates 1 and 2 , which have the highest $M R$. For decreasing $M R$ the mixing model becomes more and more accurate.

A similar approach was applied to evaluate the accuracy of the modeled $\epsilon$ ", given the accuracy of the characterization 
TABLE IV

ACCURACY OF THE MODEL PREDICTIONS VERSUS TOLERANCE IN EXTRACTED $\epsilon^{\prime}$ DUE TO UNCERTAINTY IN PATCH LENGTH OF +/- 0.5MM

\begin{tabular}{r|cc|ccc}
\hline \hline & \multicolumn{2}{|c|}{ Model accuracy } & \multicolumn{3}{|c}{ Accuracy characterization method } \\
\hline \hline & $\overline{\Delta \epsilon^{\prime}}$ & $S D$ & $\epsilon^{\prime}$ & $\Delta \epsilon_{L+0.5 \mathrm{~mm}}^{\prime}$ & $\Delta \epsilon_{L-0.5 \mathrm{~mm}}^{\prime}$ \\
\hline \hline fabric 1 & 0.061 & 0.040 & 1.767 & 0.039 & 0.044 \\
\hline fabric 2 & 0.074 & 0.053 & 1.870 & 0.050 & 0.040 \\
\hline fabric 3 & 0.024 & 0.018 & 1.942 & 0.049 & 0.055 \\
\hline fabric 4 & 0.014 & 0.008 & 1.849 & 0.047 & 0.043 \\
\hline foam & 0.006 & 0.005 & 1.2368 & 0.048 & 0.086 \\
\hline fabric 5 & 0.002 & 0.002 & 1.173 & 0.022 & 0.023 \\
\hline \hline
\end{tabular}

TABLE V

ACCURACY OF THE MODEL PREDICTIONS VERSUS TOLERANCE IN EXTRACTED $\epsilon$ " DUE TO UNCERTAINTY IN MEASURED ANTENNA $e_{c d}$ OF $+/$ $5 \%$

\begin{tabular}{r|cc|ccr}
\hline \hline & \multicolumn{2}{|c|}{ Model accuracy } & \multicolumn{3}{|c}{ Accuracy characterization method } \\
\hline \hline & $\overline{\Delta \epsilon "}$ & SD & $\epsilon "$ & $\Delta \epsilon^{\prime \prime} e_{c d}-5 \%$ & $\Delta \epsilon^{\prime \prime} e_{c d}+5 \%$ \\
\hline \hline fabric 1 & 0.029 & 0.022 & 0.0742 & 0.0036 & 0.0006 \\
\hline fabric 2 & 0.038 & 0.031 & 0.0937 & 0.0126 & 0.0039 \\
\hline fabric 3 & 0.009 & 0.007 & 0.0560 & 0.0081 & 0.0003 \\
\hline fabric 4 & 0.005 & 0.005 & 0.0302 & 0.0027 & 0.0043 \\
\hline foam & 0.001 & 0.001 & 0.0263 & 0.0043 & 0.0035 \\
\hline fabric 5 & 0.0003 & 0.0005 & 0.0032 & 0.0021 & 0.0032 \\
\hline \hline
\end{tabular}

method. We extracted $\epsilon$ " for all substrate materials at $R H=$ $40 \%$ and this under the assumption of an antenna efficiency measurement error of $+/-5 \%$. This approach allows us to estimate the characterization procedure's accuracy with respect to $\epsilon^{\prime \prime}$, since the substrate losses have a pronounced influence on antenna efficiency, as seen from the kriging models based on error function $M S E_{2}$. The absolute errors between extracted $\epsilon$ " for $e_{c d}-5 \%$ and $e_{c d}+5 \%$ and the nominal extracted $\epsilon "$ at $R H=40 \%$ are denoted by $\Delta \epsilon{ }^{\prime \prime} e_{c d}-5 \%$ and $\Delta \epsilon " e_{c d}+5 \%$, respectively and are also listed in Table V. Similar to the real part of $\epsilon$, the error in modeled $\epsilon$ " is comparable with the estimated accuracy of the characterization procedure for the substrates exhibiting a high $M R$, whereas for the substrates with low losses and low $M R$, the accuracy of the modeled $\epsilon^{\prime}$ increases.

\section{CONCLUSions}

In this paper, a novel and dedicated characterization approach for determining the constitutive properties of all materials comprised in textile antenna design was proposed and this for altering environmental conditions. The characterization technique has the advantage of being simple in sample preparation compared to resonator cavity perturbation methods or transmission line methods. Only two reflection coefficient measurements are required, yielding small measurement times. Moreover, the surrogate-based optimization approach is efficient in terms of number of sample evaluations and provides an accurate solution to the inverse problem. Errors in extracted electromagnetic properties result from simplifications in the simulation model and measurement errors. In particular, discarding the SMA connector in the simulation model results in an uncertainty in measured reflection coefficient. This has an influence on how well measured bandwidth relates to the substrate losses. In particular, low loss substrates are more sensitive to this simplification of the simulation model, compared to substrates exhibiting high dielectric losses. A combination of two antenna figures of merit, being antenna efficiency and antenna reflection coefficient, makes the characterization process less sensitive to measurement errors that relate dielectric losses with measured antenna performances. In addition, a dielectric mixing model based upon the volumetric fraction of the absorbed moisture in the substrates was developed. It is shown that the accuracy of the modeled complex permittivity of the fabric substrates exhibiting a high moisture regain is comparable with the estimated accuracy of the characterization procedure. For the substrates with a low $M R$, the accuracy of the dielectric mixing model increases.

\section{REFERENCES}

[1] P. Massey, "Mobile phone fabric antennas integrated within clothing," in Antennas and Propagation, 2001. Eleventh International Conference on (IEE Conf. Publ. No. 480), vol. 1, 2001.

[2] P. Salonen and L. Hurme, "A novel fabric WLAN antenna for wearable applications," in Antennas and Propagation Society International Symposium, 2003. IEEE, vol. 2, june 2003, pp. 700-703.

[3] A. Tronquo, H. Rogier, C. Hertleer, and L. V. Langenhove, "Robust planar textile antenna for wireless body LANs operating in $2.45 \mathrm{GHz}$ ISM band," IEE Electronics Letters, vol. 42, no. 3, pp. 142-143, February. 2006.

[4] M. Klemm, I. Locher, and G. Tröster, "A novel circularly polarized textile antenna for wearable applications," in Microwave Conference, 2004. 34th European, vol. 1, oct. 2004, pp. 137-140.

[5] T. Kennedy, P. Fink, A. Chu, N. Champagne, G. Lin, and M. Khayat, "Body-worn e-textile antennas: The good, the low-mass, and the conformal," Antennas and Propagation, IEEE Transactions on, vol. 57, no. 4, pp. 910-918, april 2009.

[6] C. Hertleer, H. Rogier, L. Vallozzi, and L. Van Langenhove, "A textile antenna for off-body communication integrated into protective clothing for fire-fighters," IEEE Trans. Antennas Propag., Special Issue on Antennas and Propagation for Body-Centric Wireless Communications, vol. 57, no. 4, Part 1, pp. 919-925, Apr. 2009.

[7] L. Vallozzi, H. Rogier, and C. Hertleer, "Dual polarized textile patch antenna for integration into protective garments," Antennas and Wireless Propagation Letters, IEEE, vol. 7, pp. 440-443, 2008.

[8] M. L. Scarpello, I. Kazani, C. Hertleer, H. Rogier, and D. Vande Ginste, "Stability and efficiency of screen printed wearable and washable antennas," Antennas and Wireless Propagation Letters, IEEE, vol. PP, no. 99 , p. 1, 2012.

[9] A. Dierck, F. Declercq, and H. Rogier, "Review of active textile antenna co-design and optimization strategies," in 2011 IEEE international conference on RFID-technologies and applications, Proceedings. IEEE, 2011, pp. 194-201.

[10] D. Cottet, J. Gryzb, T. Kirstein, and G. Tröster, "Electrical characterization of textile transmission lines," IEEE Trans. on Advanced Packaging, vol. 26, no. 2, pp. 182-190, May. 2003.

[11] J. Lilja and P. Salonen, "Textile material characterization for softwear antennas," in Proceedings of the 28th IEEE conference on Military communications, Piscataway, NJ, USA, 2009, pp. 628-634.

[12] F. Declercq, H. Rogier, and C. Hertleer, "Permittivity and loss tangent characterization for garment antennas based on a new matrix-pencil twoline method," Antennas Propag., IEEE Trans., vol. 56, no. 8, pp. 25482554, Aug. 2008.

[13] L. F. Chen, C. K. Ong, C. P. Neo, V. V. Varadan, and V. K. Varadan, Microwave electronics: Measurements and Materials Characterization, 1st ed. Chichester, West Sussex P019 8SQ U.K.: John Wiley \& Sons Ltd, 2004.

[14] C. Hertleer, A. Van Laere, H. Rogier, and L. Van Langenhove, "Influence of relative humiditiy on textile antenna performance," Tex Res J, vol. 2, pp. 177-183, 2010. 
[15] S. Sankaralingam and B. Gupta, "Determination of dielectric constant of fabric materials and their use as substrates for design and development of antennas for wearable applications," Instrumentation and Measurement, IEEE Transactions on, vol. 59, no. 12, pp. 3122-3130, Dec. 2010.

[16] R. Shaw, B. Long, D. Werner, and A. Gavrin, "The characterization of conductive textile materials intended for radio frequency applications," Antennas and Propagation Magazine, IEEE, vol. 49, no. 3, pp. 2-40, Jun. 2007.

[17] O. Yuehui and W. Chappell, "High frequency properties of electrotextiles for wearable antenna applications," Antennas Propag., IEEE Trans., vol. 56, no. 2, pp. 381-389, Feb. 2008.

[18] J. Lilja, P. Salonen, and P. de Maagt, "Characterization of conductive textile materials for softwear antenna," in Antennas and Propagation Society International Symposium, 2009. IEEE, Jun. 2009, pp. 1-4.

[19] — - "Environmental characterization of industrial fabric for softwear antenna," in Antennas and Propagation Society International Symposium, 2009. IEEE, Jun. 2009, pp. 1-4.

[20] K. Bal and V. K. Kothari, "Measurement of dielectric propeties of textile materials and their applications," Indian Journal of Fibre and Textile Research, vol. 34, pp. 191-199, Jun 2009.

[21] T. Kaija, J. Lilja, and P. Salonen, "Exposing textile antennas for harsh environment," in Military Communications Conference, 2010 - MILCOM 2010, 31 2010-nov. 3 2010, pp. 737-742.

[22] I. Couckuyt, F. Declercq, T. Dhaene, H. Rogier, and L. Knockaert, "Surrogate-based infill optimization applied to electromagnetic problems," Int. J. RF Microw. Comput.-Aided Eng., vol. 20, no. 5, pp. 492 501, Sept. 2010.

[23] J. Zhang, M. Koledintseva, J. Drewniak, D. Pommerenke, R. DuBroff, Z. Yang, W. Cheng, K. Rozanov, G. Antonini, and A. Orlandi, "Reconstruction of dispersive dielectric properties for PCB substrates using a genetic algorithm," Electromagnetic Compatibility, IEEE Transactions on, vol. 50, no. 3, pp. 704-714, Aug. 2008.

[24] E. Kilic, U. Siart, and T. Eibert, "Regularized 1-D dielectric profile inversion in a uniform metallic waveguide by measurement and simulation," Microwave Theory and Techniques, IEEE Transactions on, vol. 60, no. 5, pp. 1437-1443, May 2012.

[25] D. Popovic, L. McCartney, C. Beasley, M. Lazebnik, M. Okoniewski, S. Hagness, and J. Booske, "Precision open-ended coaxial probes for in vivo and ex vivo dielectric spectroscopy of biological tissues at microwave frequencies," Microwave Theory and Techniques, IEEE Transactions on, vol. 53, no. 5, pp. 1713-1722, May 2005.

[26] S. Lambot, E. Slob, I. Van Den Bosch, B. Stockbroeckx, and M. Vanclooster, "Modeling of ground-penetrating radar for accurate characterization of subsurface electric properties," Geoscience and Remote Sensing, IEEE Transactions on, vol. 42, no. 11, pp. 2555-2568, Nov. 2004.

[27] I. Rekanos, "Neural-network-based inverse-scattering technique for online microwave medical imaging," Magnetics, IEEE Transactions on vol. 38, no. 2, pp. 1061-1064, Mar 2002.

[28] E. Bermani, S. Caorsi, and M. Raffetto, "Microwave detection and dielectric characterization of cylindrical objects from amplitude-only data by means of neural networks," Antennas and Propagation, IEEE Transactions on, vol. 50, no. 9, pp. 1309-1314, Sep 2002.

[29] M. Simsek and N. Tezel, "The reconstruction of shape and impedance exploiting space mapping with inverse difference method," Antennas and Propagation, IEEE Transactions on, vol. 60, no. 4, pp. 1868-1877, April 2012.

[30] K. Bal and V. K. Kothari, "Permittivity of woven fabrics: A comparison of dielectric formulas for air-fiber mixture," Dielectrics and Electrical Insulation, IEEE Transactions on, vol. 17, no. 3, pp. 881-889, June 2010.

[31] M. Dobson, F. Ulaby, M. Hallikainen, and M. El-Rayes, "Microwave dielectric behavior of wet soil-part ii: Dielectric mixing models," Geoscience and Remote Sensing, IEEE Transactions on, vol. GE-23, no. 1, pp. 35-46, Jan. 1985.

[32] F. Bouttout, F. Benabdelaziz, A. Benghalia, D. Khedrouche, and T. Fortaki, "Uniaxially anisotropic effects on resonance of rectangular microstrip patch antenna," IEE Electronics Letters, vol. 35, no. 4, pp. 255 256, February. 1999.

[33] "Theory of operation for momentum," Agilent Technologies. [Online] Available: http://edocs.soco.agilent.com

[34] D. R. Jones, M. Schonlau, and W. J. Welch, "Efficient global optimization of expensive black-box functions," Journal of Global Optimization, vol. 13, no. 4, pp. 455-492, 1998.

[35] J. P. C. Kleijnen, W. van Beers, and I. van Nieuwenhuyse, "Expected improvement in efficient global optimization through bootstrapped krig ing," Journal of global optimization, vol. 54, no. 1, pp. 59-73, Sept. 2012.

[36] Rogers Corporation, "RO4000 series high frequency circuit materials." [Online]. Available: http://www.rogerscorp.com/documents/726/acm/RO4000-Laminates

[37] [Online]. Available: http://sumo.intec.ugent.be

[38] [Online]. Available: http://www.lessemf.com/1212.pdf

[39] P. Barthia, I. Bahl, R. Garg, and A. Ittipiboon, Microstrip Antenna Design Handbook. Artech House Publishers., 1987.

[40] R. Johnston and J. McRory, "An improved small antenna radiationefficiency measurement method," Antennas Propag. Mag., IEEE, vol. 40, no. 5, pp. 40-48, oct 1998.

[41] R. Buchner, J. Barthel, and J. Stauber, "The dielectric relaxation of water between $0^{\circ} \mathrm{C}$ and $35^{\circ} \mathrm{C}$," Chemical Physics Letters, vol. 306, no. 1-2, pp. $57-63,1999$.

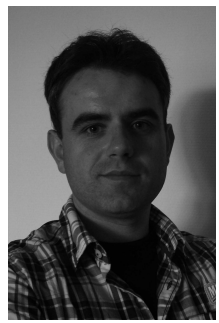

Frederick Declercq received his M.Sc. degree in electronic engineering from Howest, university college West Flanders, Kortrijk, Belgium. He received the Ph.D. degree in electrical engineering at the faculty of engineering at Ghent University, Ghent, Belgium, in 2011. Since September 2005, he has been with the electromagnetics group, department of Information Technology (INTEC) at Ghent University. $\mathrm{He}$ is currently conducting his postdoctoral research at IMEC. His research interests are electromagnetic characterization of textile materials and flexible foam materials, the design of wearable active textile antennas and energy scavenging techniques for wireless sensor nodes applied in body area networks.

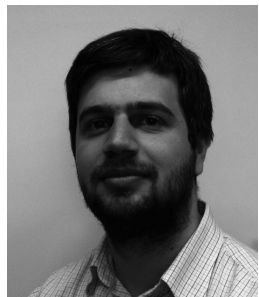

Ivo Couckuyt received his M.Sc. degree in Computer Science from the University of Antwerp (UA) in 2007. He received his Ph.D. degree in Engineering from Ghent University, Ghent, Belgium, in 2013 Starting from June 2013 he is active as a postdoctoral fellow in the INTEC Broadband Communication Networks (IBCN) research group of the Department of Information Technology (INTEC), Ghent University, Belgium. His research activities include machine learning, bioinformatics, optimal design, numerical analysis techniques, surrogate modeling, surrogate-based optimization and inverse modeling of time-consuming problems.

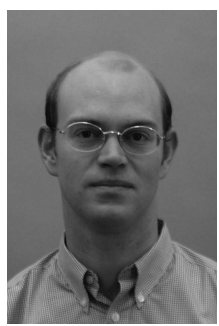

Hendrik Rogier was born in 1971 . He received the Electrical Engineering and the Ph.D. degrees from Ghent University, Gent, Belgium, in 1994 and in 1999, respectively. He is currently a Full Professor with the Department of Information Technology. From October 2003 to April 2004, he was a Visiting Scientist at the Mobile Communications Group of Vienna University of Technology. He authored and coauthored about 80 papers in international journals and about 100 contributions in conference proceedings. He is serving as a member of the Editorial Boarding of IET Science, Measurement Technology and acts as the URSI Commission B representative for Belgium. His current research interests are the analysis of electromagnetic waveguides, electromagnetic simulation techniques applied to electromagnetic compatibility (EMC) and signal integrity (SI) problems, as well as to indoor propagation and antenna design, and in smart antenna systems for wireless networks. Dr. Rogier was twice awarded the URSI Young Scientist Award, at the 2001 URSI Symposium on Electromagnetic Theory and at the 2002 URSI General Assembly. He is a Senior Member of the IEEE. 


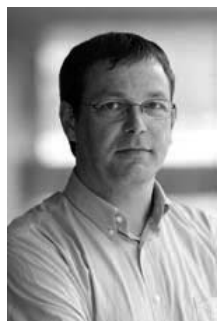

Tom Dhaene received the Ph.D. degree in electrotechnical engineering from Ghent University, Ghent, Belgium, in 1993. In 1993, he joined the EDA company Alphabit (now part of Agilent). He was one of the key developers of the planar EM simulator ADS Momentum, ADS Model Composer, and ADS Broadband SPICE. Since 2007, he has been a Full Professor with the Department of Information Technology (INTEC), Ghent University - iMINDS He has authored or coauthored more than 250 peerreviewed papers and abstracts in international conference proceedings, journals, and books. He is the holder of 5 U.S. patents. 